\title{
A fast method to estimate body capacitance to ground at mid frequencies
}

\author{
Carles Aliau-Bonet ${ }^{1}$ and Ramon Pallas-Areny ${ }^{1,2}$ \\ 1. Instrumentation, Sensors and Interfaces Group, Department of Electronic Engineering, Universat Politècnica de Catalunya (UPC, \\ BarcelonaTech), Castelldefels (Barcelona), Spain \\ 2. E-mail any correspondence to: ramon.pallas@upc.edu
}

\begin{abstract}
Impedance measurements that involve the human body are affected by the capacitance between the body and earth ground. This paper describes a fast method to estimate that capacitance at $10 \mathrm{kHz}$, which is valid for impedance analyzers intended to measure ungrounded impedances. The method does not require any external component other than two common capacitors and two conductive electrodes in contact with the body.
\end{abstract}

Keywords: Human body capacitance, stray capacitance, impedance measurement error

\section{Introduction}

The stray capacitance between the human body and earth ground can play a significant role in bioimpedance measurement systems where it may yield, for example, apparent inductive components not associated to any magnetic phenomena $[1,2]$. It is also an intervening factor in the assessment of human body impedance for risk analysis in case of electric contacts [3] or electromagnetic radiation [4] and in electrostatic discharge studies [5], and has strong influence in power-line interference in biopotential measurements [6-8]. Finally, changes due to body capacitance are the sensing principle of capacitive tactile, proximity [9] and seat occupancy sensors [10].

Body capacitance to ground can be measured by using a grounded oscilloscope and a passive 10:1 voltage divider probe $[6,7]$, or a variable resistor, an amplifier and a voltmeter with grounded reference terminal [8], that estimate the power line $(50 / 60 \mathrm{~Hz})$ current through the body. These methods, however, heavily depend on the strength of existing power line fields and on the resistance of the contact with the body, hence on contact pressure. Some floating (battery-supplied) LCR meters can also measure that capacitance by connecting one of their input terminals to earth ground, but some of them can measure only up to $10 \mathrm{kHz}$, yet other cannot measure capacitances below, say, $100 \mathrm{pF}$.

Impedance meters/analyzers that have their signal ground at earth potential and are not battery-supplied cannot directly measure grounded impedances such as body-to-earth capacitance unless provided with special probes [11]. This shortcoming can be overcome by applying the method described in [12] wherein a network is built that comprises two resistors connected in series and the human body is connected to the node common to both resistors. The other end of each resistor is connected to a respective terminal of the impedance analyzer. The measured impedance comprises a real part, due to the resistances, and a positive (hence inductive) imaginary part that is proportional to the product of the two resistances, the measurement frequency and the capacitance from the body to ground. Since the resistances of the two resistors and the measurement frequency are known, it is straightforward to calculate the capacitance to ground from the measured imaginary part. This method works fine from $50 \mathrm{kHz}$ to $1 \mathrm{MHz}$ but below $50 \mathrm{kHz}$ that imaginary impedance component is too small compared to the real component and the limited resolution of the meter yields erratic readings.

In this paper, we propose to estimate body capacitance to ground by evaluating the effect of that capacitance when measuring two known capacitances connected in series. First, that effect is qualitatively analyzed and then the proposed method is described and its performance assessed.

\section{Effect of stray capacitances to ground in auto-balancing impedance bridges}

Most commercial impedance analyzers are nowadays based on auto-balancing bridges [11]. Figure 1 shows the equivalent circuit when impedance $Z_{\mathrm{b}}$ is measured by connecting it between terminals $\mathrm{H}$ and $\mathrm{L}$. The instrument applies a voltage to terminal $\mathrm{H}$ and measures the current at node $\mathrm{L}$, which is held at $0 \mathrm{~V}$ (virtual ground) by a feedback loop. This current measurement method is analogous to a transimpedance amplifier based on an ideal op amp [11, 13]. The impedance at a given frequency is calculated by dividing the vector voltage (phasor) $V_{\mathrm{HG}}$ measured between $\mathrm{H}$ and ground by the vector current (phasor) $I_{\mathrm{L}}$ measured at that frequency.

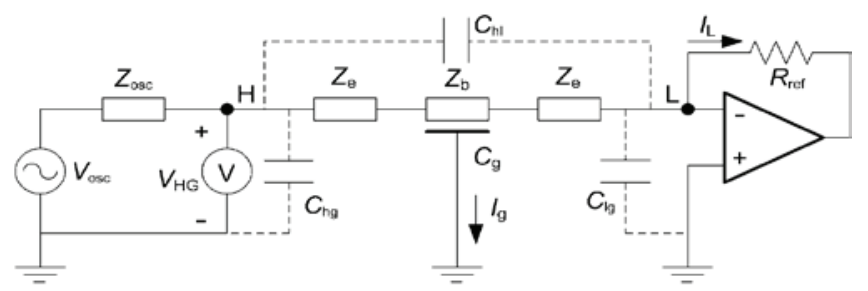

Fig.1: Equivalent circuit for impedance measurements based on an auto-balancing bridge.

Measurement uncertainty depends on [14]: (1) contact impedance $Z_{\mathrm{e}}$ between the measured material and instrument terminals $\mathrm{H}$ and $\mathrm{L}$ due to cables and electrodes; 
(2) leakage ("air") capacitance $C_{\mathrm{hl}}$ between $\mathrm{H}$ and $\mathrm{L}$; and (3) parasitic capacitances $C_{\mathrm{hg}}, C_{\mathrm{lg}}$ between each electrode and ground and $C_{\mathrm{g}}$ between the measured material and ground.

This measurement method is immune to $C_{\mathrm{hg}}$ and $C_{\mathrm{lg}}$ [15], which has contributed to its popularity, but cannot measure grounded impedances [11]. Moreover, the "air" capacitance $C_{\mathrm{hl}}$ between electrodes and the capacitance $C_{\mathrm{g}}$ from the material to ground affect the measurement results [13]. $C_{\mathrm{hl}}$ mostly depends on interelectrode distance and can be minimized by placing a small grounded electric shield between the electrodes [11]. $C_{\mathrm{g}}$, however, cannot be easily minimized because it is a distributed capacitance, hence it will depend on the volume of the material and its distance to ground. Furthermore, $C_{\mathrm{g}}$ increases when a grounded electric shield is placed between electrodes to reduce $C_{\mathrm{hl}}$ [13].

Displacement current $I_{\mathrm{g}}$ in figure 1 reduces the measured current $I_{\mathrm{L}}$ hence the calculated impedance will be larger than the expected $2 Z_{\mathrm{e}}+Z_{\mathrm{b}}$. When the human body impedance is measured, $C_{\mathrm{g}}$ can be sizeable because of the large volume of the body and because the distance to ground can be as short as the thickness of footwear soles. The relevant effects of $C_{\mathrm{g}}$ in impedance measurements, either bipolar with direct contact [13] or capacitive electrodes [16], or tetrapolar [10] warrant the interest of its measurement.

\section{Measurement method}

In a scenario where bipolar impedances are measured on the human body, we propose to estimate the capacitance from the body to ground by connecting a known capacitor between each electrode and the impedance analyzer. Figure 2 shows the resulting equivalent circuit. If the capacitance of the added capacitors $C$ is small enough for its impedance to be much larger than that of the body and the electrodes at the measurement frequency,

$$
\frac{1}{j \omega C / 2}>>2 Z_{\mathrm{e}}+Z_{\mathrm{b}},
$$

then the equivalent circuit can be simplified into that in figure 3 .

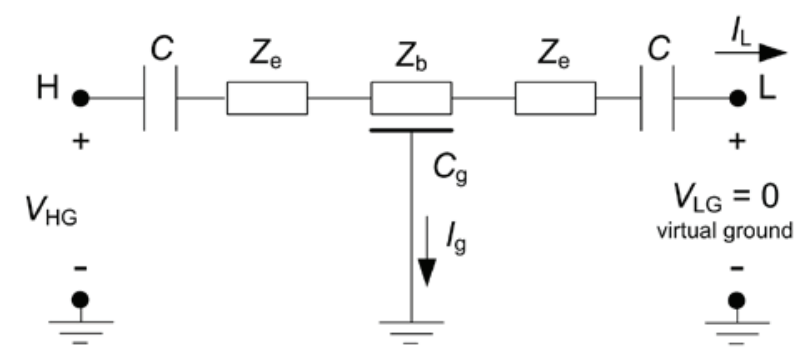

Fig.2: Equivalent circuit when a capacitor $C$ is connected in series with each electrode in bipolar body impedance measurements.

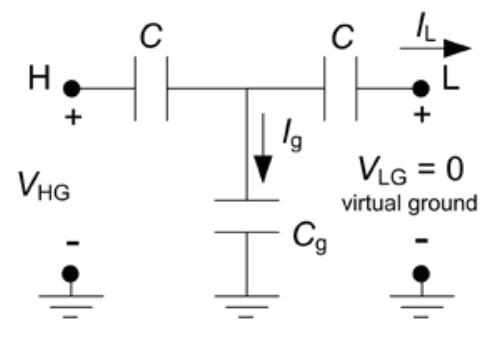

Fig.3: Simplified equivalent circuit when $C$ is small enough for its impedance to predominate over that of the electrodes and the body at the measurement frequency.

By applying the star-delta impedance transformation, the impedance between $\mathrm{H}$ and $\mathrm{L}$ in figure 3, as calculated by the impedance analyzer, will be

$$
Z_{\mathrm{HL}}(\mathrm{j} \omega)=\frac{V_{\mathrm{HG}}}{I_{\mathrm{L}}}=\frac{1}{\mathrm{j} \omega C}+\frac{1}{\mathrm{j} \omega C}+\frac{\frac{1}{\mathrm{j} \omega C} \frac{1}{\mathrm{j} \omega C}}{\frac{1}{\mathrm{j} \omega C_{\mathrm{g}}}}=\frac{1}{\mathrm{j} \omega \frac{C}{2+C_{\mathrm{g}} / C}}
$$

and the equivalent capacitance,

$$
C_{\mathrm{HL}}=\frac{1}{\mathrm{j} \omega Z_{\mathrm{HL}}}=\frac{C}{2+C_{\mathrm{g}} / C} .
$$

When $C_{\mathrm{g}}=0$, we have $C_{\mathrm{HL}}=C / 2$ but otherwise the measured capacitance will be smaller than $C / 2$. Solving (3) for $C_{\mathrm{g}}$ we obtain

$$
C_{\mathrm{g}}=C\left(\frac{C}{C_{\mathrm{HL}}}-2\right) \text {. }
$$

Therefore, selecting $C$ such that at the desired measurement frequency the condition (1) is fulfilled, $C_{\mathrm{g}}$ can be estimated from (4). Usually, selecting $C$ within the range of the expected value for $C_{\mathrm{g}}$ is a good choice.

\section{Experimental design}

The proposed method to estimate $C_{\mathrm{g}}$ has been applied in two different instruments based on auto-balancing bridges: an impedance analyzer (Agilent 4294A) and a handheld LCR meter (Motech MT4080). The relative uncertainty of the $4294 \mathrm{~A}$ when measuring capacitances between $10 \mathrm{pF}$ to $100 \mathrm{pF}$ at $10 \mathrm{kHz}$ ranges from $\pm 1 \%$ to $\pm 0.3 \%$ of the reading [17], whereas that of the MT4080 when measuring capacitances between $15.91 \mathrm{pF}$ and $159.1 \mathrm{pF}$ at $10 \mathrm{kHz}$ is $\pm 0.5 \%$ of the reading plus \pm 1 [18]. The $4294 \mathrm{~A}$ is a toprange impedance analyzer that cannot measure grounded impedances [11] whereas the MT4080 is a low-cost LCR meter that can measure grounded impedances because it is supplied by batteries, hence has floating inputs. Nevertheless, it can measure only up to $10 \mathrm{kHz}$. In order for the electromagnetic environment to be similar for both 
instruments, the guard terminal of the MT4080, which is connected to its (floating) signal ground [18], has been connected to earth ground.

Four volunteers (two men, subjects \#1 and \#2, and two women, subjects \#3 and \#4) have been measured. Their basic anthropometric data and shoes' outsole and type are given in Table 1. BMI is the body-mass index calculated by dividing the mass $(\mathrm{kg})$ by the squared height $\left(\mathrm{m}^{2}\right)$.

Table 1. Data from the measured subjects. BMI is the body mass index $\left(\mathrm{kg} / \mathrm{m}^{2}\right)$.

\begin{tabular}{ccccl}
\hline Subject & Height $(\mathrm{m})$ & Weight $(\mathrm{kg})$ & BMI & \multicolumn{1}{c}{ Shoes' outsole and type } \\
\hline 1 & 1.90 & 130 & 36 & Rubber, sports shoes \\
2 & 1.80 & 90 & 28 & Leather dress shoes \\
3 & 1.66 & 65 & 24 & Rubber, boots with $4 \mathrm{~cm}$ heel \\
4 & 1.50 & 42 & 19 & Leather, boot with $7 \mathrm{~cm}$ heel
\end{tabular}

Subjects were successively connected to the impedance meters by two $4.9 \mathrm{~cm}^{2}$ brass plates and $12 \mathrm{~cm}$ long braided wire to minimize the serial inductance of the connection. A respective ceramic $68 \mathrm{pF}$ capacitor $( \pm 10 \%$ tolerance $)$ was soldered to the end of each cable. The actual values of the capacitors at $10 \mathrm{kHz}$, measured with the 4294A, were $74.1 \mathrm{pF}$ for the one connected to terminal $\mathrm{H}$ and $74.0 \mathrm{pF}$ for the one connected to terminal $\mathrm{L}$. These values guarantee a high-enough impedance for $C$ at $10 \mathrm{kHz}$ to fulfill equation (1) as the body impedance at this frequency is about $499 \Omega$ in series with $62 \mathrm{nF}$ and the electrodes have yet smaller impedance [19].

The measurement protocol was as follows: the subject was seated on an office chair with plastic caster wheels, and held a (dry) electrode between the thumb and index finger of each hand. The hands were about $20 \mathrm{~cm}$ apart and rested on the wooden surface of the table that held the impedance meter. This separation guaranteed a small-enough leakage (air) capacitance between electrodes. Increasing that separation could be counterproductive as the cables to connect the electrodes to the meter should then be longer, which would increase their inductance. Each subject was measured twice: first with the feet at about $10 \mathrm{~cm}$ from ground and later with the shoes in direct contact with the tiled floor.

\section{Results}

Table 2 shows the results obtained with the 4294A and Table 3 those obtained with the MT4080.

Table 2. Capacitance $C_{\mathrm{HL}}$ measured with the 4294A impedance analyzer and calculated body capacitance to ground $C_{\mathrm{g}}$ for the four subjects and two feet heights above to ground: $10 \mathrm{~cm}(\mathrm{u})$ and direct outsole contact (d).

\begin{tabular}{ccccc}
\hline Subject & $C_{\mathrm{HLu}}(\mathrm{pF})$ & $C_{\mathrm{gu}}(\mathrm{pF})$ & $C_{\mathrm{HLd}}(\mathrm{pF})$ & $C_{\mathrm{gd}}(\mathrm{pF})$ \\
\hline 1 & 22.5 & 95.4 & 18.2 & 153.0 \\
2 & 23.5 & 85.1 & 17.8 & 159.7 \\
3 & 24.8 & 72.9 & 20.5 & 119.2 \\
4 & 25.5 & 66.8 & 20.2 & 123.2
\end{tabular}

$C_{\mathrm{HL}}$ is the capacitance measured by each instrument at $10 \mathrm{kHz}$ and $C_{\mathrm{g}}$ is the capacitance calculated from (4) for the two feet positions ( $u$ : up, d: down). No effect of the contact pressure with the electrodes was noticed.

Table 3. Capacitance $C_{\mathrm{HL}}$ measured with the MT4080 LCR meter and calculated body capacitance to ground $C_{\mathrm{g}}$ for the four subjects and two feet heights above ground: $10 \mathrm{~cm}(\mathrm{u})$ and direct outsole contact (d).

\begin{tabular}{ccccc}
\hline Subject & $C_{\mathrm{HLu}}(\mathrm{pF})$ & $C_{\mathrm{gu}}(\mathrm{pF})$ & $C_{\mathrm{HLd}}(\mathrm{pF})$ & $C_{\mathrm{gd}}(\mathrm{pF})$ \\
\hline 1 & 22.4 & 96.5 & 18.0 & 156.3 \\
2 & 23.4 & 86.1 & 17.8 & 159.7 \\
3 & 25.0 & 71.1 & 20.2 & 123.2 \\
4 & 25.8 & 64.3 & 19.8 & 128.6
\end{tabular}

\section{Discussion}

$C_{\mathrm{g}}$ values display the same trends for all subjects and both instruments. For any given subject, the body capacitance to ground increases when the feet rest on ground, i.e. the distance from the body to ground decreases, regardless of the impedance meter used. That increase is larger for subjects \#2 and \#4 as expected from the thinner leather outsoles they wore with respect to subjects \#1 and \#3. This is in agreement with the results obtained with the more involved method to estimate $C_{\mathrm{g}}$ described in [12] that used a circuit similar to that in figure 2 but with two resistors instead of the two capacitors $C$.

For a given feet position, $C_{\mathrm{g}}$ values increase with BMI as it could be expected, but this dependence on BMI is far smaller than that on the distance from feet to ground. In Table 2, for example, $C_{\mathrm{g}}$ for subject \#2 with feet on ground is larger than that for subject \#1 in spite of the larger BMI of the first, and $C_{\mathrm{g}}$ for subject \#4 with feet on ground is about $30 \%$ larger than that for subject \#1 with feet about $10 \mathrm{~cm}$ above ground, in spite that BMI for this one is 36 as compared to only 19 for subject \#4. Therefore, closeness to grounded objects is more relevant than body size, and makes footwear a factor to account for in body impedance measurement and in electromagnetic hazard analysis.

The differences between $C_{\mathrm{HL}}$ values measured in the same situation by each instrument range from $0.2 \mathrm{pF}$ to $0.5 \mathrm{pF}$. However, the corresponding difference between calculated $C_{\mathrm{g}}$ values is a bit larger than $5 \mathrm{pF}$. This is a consequence of the nonlinear relationship between both capacitances, as shown in (4), but in any case, the deviation is just around $4 \%$, which is quite acceptable.

Calculated $C_{\mathrm{g}}$ values at $100 \mathrm{kHz}$ for subject \#1 in [12] were $96.6 \mathrm{pF}$ with raised feet and $158.1 \mathrm{pF}$ with feet on ground. These values are very close to the corresponding ones at $10 \mathrm{kHz}$ in Table 2 and corroborate the validity of the method. Further, whereas figure 8 in [12] showed some erratic tendencies below $50 \mathrm{kHz}$, no reading fluctuations were observed here. This is because in complex impedance measurements it is easier to have good resolution in the predominant component (real or imaginary) than in the smaller quadrature component. Both here and in [12], the method relies on the "inductive component" contributed by 
$C_{\mathrm{g}}$ but whereas in [12] that component added to a large real component, due to the two series-connected resistors, here it adds to the reactance of the two capacitors $C$ and the series resistance (from the electrodes and the body) is negligible. Therefore, this novel method is clearly advantageous because it is fast and allows power-line supplied impedance analyzers to measure grounded impedances.

\section{Acknowledgements}

The authors would like to thank the Castelldefels School of Telecommunications and Aerospace Engineering (EETACUPC, BarcelonaTech) for its research facilities, Mr. F. López for his technical support, and all volunteers for their cooperation.

\section{References}

1. Gersing E, Schäffer M, Osypka M. The appearance of positive phase angles in impedance measurements on extended biological objects. Inno Tech Biol Med. 1995;16:71-76.

2. Aliau-Bonet C, Pallas-Areny R. On the effect of body capacitance to ground in tetrapolar bioimpedance measurements. IEEE Trans Biomed Eng. 2012;59:3405-3411. http://dx.doi.org/10.1109/TBME.2012.2216880

3. De Santis V, P. A. Beeckman P A, Lampasi D A, et al. Assessment of human body impedance for safety requirements against contact currents for frequencies up to $110 \mathrm{MHz}$. IEEE Trans Biomed Eng. 2011;58:390-396. http://dx.doi.org/10.1109/TBME.2010.2066273

4. Kanai H, Chatterjee I, Gandhi O P. Human Body Impedance for Electromagnetic Hazard Analysis in the VLF to MF Band. IEEE Trans Microwave Theory Techn. 1984;32:763-772. http://dx.doi.org/10.1109/TMTT.1984.1132770

5. Jonassen N. Human body capacitance: Static or dynamic concept? In Proc. [ESD] Electr Overstress /Electrost Disch Symp., Oct- 6-8. 1998;111-117.

6. Pallas-Areny R, Colominas J. Simple, Fast Method for Patient Body Capacitance and Power-line Electric Interference Measurement. Med Biol Eng Comput. 1991;39:561-563. http://dx.doi.org/10.1007/BF02442332

7. Serrano R E, Gasulla M, Casas O, Pallas-Areny R. Power Line Interference in Ambulatory Biopotential Measurements. In Proc. 25th Ann Intl Conf Eng Med Biol Soc., Cancun (México). 2003;3024-3027. http://dx.doi.org/10.1109/iembs.2003.1280777
8. Haberman M, Cassino A, Spinelli E. Estimation of stray coupling capacitances in biopotential measurements. Med Biol Eng Comput. 2011;49:1067-1071. http://dx.doi.org/10.1007/s11517-011-0811-6

9. Buller W, Wilson B Measurement and Modeling Mutual Capacitance of Electrical Wiring and Humans. IEEE Trans Instrum Meas. 2006;55:1519-22. http://dx.doi.org/10.1109/TIM.2006.880293

10. George B, Zangl H, Bretterklieber T, et al. Seat occupancy detection based on capacitive sensing. IEEE Trans Instrum Meas. 2009;58:1487-1494. http://dx.doi.org/10.1109/TIM.2009.2009411

11. Agilent (2006). The Impedance Measurement Handbook, A Guide to Measurement Technology and Techniques. Available from http://cp.literature.agilent.com.

12. Aliau-Bonet C, Pallas-Areny R. A novel method to estimate body capacitance to ground at mid frequencies. IEEE Trans Instrum Meas. 2013;62:2519-2525. http://dx.doi.org/10.1109/TIM.2013.2258240

13. Aliau-Bonet, C., Pallas-Areny, R. Effects of stray capacitance to ground in bipolar material impedance measurements based on direct-contact electrodes. IEEE Trans Instrum Meas. 2014;63, 2414-2421. http://dx.doi.org/10.1109/tim.2014.2310033

14. Kibble B, Williams J, Henderson L, et al. A guide to measuring resistance and impedance below $1 \mathrm{MHz}$. The Institute of Measurement and Control (NPL) London, 1999.

15. Yang W. A self-balancing circuit to measure capacitance and loss conductance for industrial transducer applications. IEEE Trans Instrum Meas. 1996;45:955-958. http://dx.doi.org/10.1109/19.543992

16. Aliau-Bonet, C., Pallas-Areny, R. Effects of stray capacitance to ground in bipolar water impedance measurements based on capacitive electrodes. XXI IMEKO World Congress, Prague, Czech Republic, August 30-September 4. 2015;676-679.

17. Agilent 4294A Precision Impedance Analyzer Data Sheet. Agilent Technologies 2008. Available from http://cp.literature.agilent.com.

18. Motech MT4080 LCR Meter Operating Manual. Motech Industries. Available from http://www.motech.com.

19. Aliau-Bonet C, Pallas-Areny R. A fast method to estimate body capacitance to ground. In Proc. XX IMEKO World Congress, Busan, Korea. 2012, Paper 990. 